\title{
Avaliação Comportamental por Meio de Triagem com a Lógica Fuzzy Para o Auxílio na Predição de Traços Esquizofrênicos em Adultos Segundo Critérios A e B do DSM-5
}

\author{
Maria Dayane Almeida Araújo ${ }^{1}$, Rhyan Ximenes de Brito ${ }^{1}$ \\ ${ }^{1}$ Instituto Federal de Educação, Ciência e Tecnologia do Ceará (IFCE) \\ Av. Tabelião Luiz Nogueira de Lima S/N - Tianguá - CE - Brazil \\ \{dayanealmeida1996, rxbrito\}@gmail.com
}

\begin{abstract}
Artificial Intelligence (AI) as a tool to support specialized dignitaries is very promising. Within this perspective, it is known that schizophrenia is a psychiatric disorder that is difficult to diagnose. In this sense, this work presents a fuzzy system whose objective is to assist professionals in the behavioral assessment of patients through the screening of characteristics that enable them to close diagnoses. The methodology was carried out based on simulations where symptoms such as delusions, hallucinations, disorganized speech among others were used as input variables. Based on the results, it can be concluded that AI has a lot to contribute in the search for solutions in the medical field, especially in the diagnosis aid.

Resumo. A Inteligência Artificial (IA) como ferramenta de apoio a dignósticos especializados mostra-se bastante promissora. Dentro dessa perspectiva sabese que a esquizofrenia é um distúrbio psiquiátrico de difícil diagnóstico. Nesse sentido este trabalho apresenta um sistema fuzzy cujo objetivo é auxiliar profissionais na avaliação comportamental de pacientes por meio da triagem de características que enseje no fechamento de diagnósticos. A metodologia foi realizada com base em simulações onde os sintomas como delírios, alucinações, discurso desorganizado entre outros foram utilizados como variáveis de entrada. Com base nos resultados pode-se concluir que a IA tem muito a contribuir na busca por soluções na área médica em especial no auxílio de diagnósticos.
\end{abstract}

\section{Introdução}

Segundo [Lobo 2018], a Inteligência Artificial (IA) é um ramo da Ciência da Computação que se propõe a desenvolver sistemas que simulem a capacidade racional humana de resolver problemas. A lógica fuzzy é uma técnica de aplicação da Inteligência Artificial que, por sua vez, tem possibilitado grandes avanços no tratamento de dados complexos.

Também conhecida como lógica multivalorada, a lógica fuzzy é uma extensão da lógica clássica. De acordo com [Marro et al. 2010] a lógica fuzzy tenta modelar o pensamento humano e a introdução dessa nova lógica tem conduzido as pesquisas para sistemas inteligentes mais adequados à realidade.

Vale salientar que o presente trabalho tem como objetivo principal, desenvolver um sistema baseado na lógica fuzzy como ferramenta para auxiliar no processo de prédiagnóstico da esquizofrenia, através de variáveis linguísticas baseadas nos critérios A 
e B do manual DSM-5, com o intuito de ajudar as pessoas acometidas por tal doença procurarem o tratamento adequado.

O presente trabalho é uma continuidade do artigo de [Araujo et al. 2019], tendo como diferencial a inclusão do critério $\mathrm{B}$ ensejando em um número maior de variáveis linguísticas, dessa forma os resultados obtidos com base nas regras de inferência tornamse mais precisos, outro diferencial foi a utilização de pesos relacionados aos níveis de gravidade dos sintomas baseados no DSM-5.

Nesses termos, é importante ressaltar que a utilização das técnicas da Inteligência Artificial, a qual possam dar respaldo a sistemas que auxiliem no processo de apoio ao diagnóstico de pessoas com esquizofrenia, tornam-se bastante relevantes dentro do contexto da informática aplicada à saúde.

Este trabalho está estruturado da seguinte forma: Na Seção 2, é apresentada a Fundamentação Teórica; na Seção 3, apresenta os Trabalhos Relacionados. Posteriormente na Seção 4, é abordada a Metodologia, em seguida, na Seção 5 são apresentados os Resultados e Discussões e, por fim, na Seção 6, são abordados as Considerações Finais.

\section{Fundamentação Teórica}

Esta seção traz um levantamento bibliográfico a respeito dos principais assuntos abordados neste trabalho.

\subsection{Lógica Nebulosa}

De acordo com [Marro et al. 2010] a lógica fuzzy, também conhecida como lógica multivalorada ou nebulosa foi introduzida em 1930, por Jan Lukasiewicz utilizando-se inicialmente de termos linguísticos, ele propôs a possibilidade de uma declaração ser verdadeira ou falsa utilizando intervalos variando entre 0 e 1. Em 1965, Lofti Zadeh publicou o artigo Fuzzy Sets que ficou conhecido como a origem da lógica fuzzy.

[Marro et al. 2010] também afirma que a lógica fuzzy tem como principal objetivo modelar o pensamento humano, tratando dados imprecisos. Diferentemente da lógica convencional, ela admite graus de pertinências, ou seja, graus de verdade.

\subsection{DSM-5 e a Esquizofrenia}

O Manual Diagnóstico e Estatístico de Transtornos Mentais (DSM-5) foi feito pela Associação Americana de Psiquiatria para definir como é feito o diagnóstico de transtornos mentais, resulta de um processo de doze anos de estudos realizados por diferentes grupos de trabalho. Tendo como objetivo final fornecer uma fonte segura e cientificamente embasada para aplicação em pesquisa e na prática clínica [Araújo and Neto 2014].

A esquizofrenia é um transtorno mental crônico caracterizada por distorções do pensamento e da percepção [Amorim et al. 2018]. Este transtorno acomente muitas pessoas, muda completamente a vida das mesmas, pois, elas criam realidades que não existem e são submetidas a um tratamento que costuma ser por toda a vida.

O tratamento envolve uma combinação de ações, tanto de cuidados especializados, quanto de cuidados da família que por sua vez é o elo que liga o paciente a nova realidade, assim como o uso de medicamentos e psicoterapia. Segundo [Rosa and Elkis ] dentro do 
tratamento é sempre válido mudar a medicação, reduzir a dosagem e utilizar medicações concomitantes para aliviar sintomas extrapiramidais.

Segundo [Behenck et al. 2011] no decorrer da doença os pacientes e seus familiares vivenciam períodos de controle e diminuição dos sintomas, alternados a momentos de recaídas que podem induzir a uma reinternação hospitalar do doente.

A psicoterapia tem como objetivo reduzir os sintomas, prevenir as recaídas e evitar a internação. No entanto, nos momentos de crise a internação deve ser encarada como uma medida protetora [Shirakawa 2000]. Dessa maneira, evidencia-se a importância da aplicação do uso de terapias, além da interação medicamentosa [Behenck et al. 2011].

\subsection{Critérios A e B Para o Diagnóstico da Esquizofrênia Segundo o DSM-5}

1. A1-Delírios: ideias ou pensamentos que não condizem com a realidade, por exemplo, o indivíduo acredita que está sendo perseguido ou acredita que alguém irá prejudica-lo [Association et al. 2014];

2. A2-Alucinações: percepções reais de objetos que não existem, por exemplo, alucinações auditivas [Association et al. 2014];

3. A3-Discurso Desorganizado: perda da ligação normal e lógica da conversa, ou seja, o discurso pode estar tão gravemente desorganizado que torna-se quase incompreensível [Association et al. 2014];

4. A4-Comportamento Grosseiramente Desorganizado ou Catatônico: pode-se manifestar de várias formas, inclusive como uma agitação imprevisível diante de determinada situação, por exemplo: dificuldades na realização das atividades cotidianas [Association et al. 2014];

5. A5-Sintomas Negativos: caracteriza-se por expressão emocional diminuída incluindo reduções nas expressões de emoções no rosto, no contato visual e outras [Association et al. 2014];

6. B-Relacionamento Interpessoal/Autocuidado: por período significativo de tempo desde o aparecimento da pertubação, o nível de funcionamento em áreas importantes do funcionamento como relacionamento interpessoal e autocuidado abaixo do nível alcançado [Association et al. 2014];

\section{Trabalhos Relacionados}

Esta seção apresenta uma revisão bibliográfica de artigos encontrados na literatura sobre a utilização da Inteligência Artificial como instrumento para auxiliar à busca por respostas a problemas relacionados a saúde, especialmente a esquizofrenia, problema tratado nesta pesquisa.

[Razzouk et al. 2000] neste artigo foram abordados os sistemas de apoio à decisão no diagnóstico de esquizofrenia que foram desenvolvidos até o momento, suas vantagens, limitações e potenciais benefícios para a prática clínica.

[Araujo et al. 2019] propôs um sistema fuzzy, objetivando auxiliar profissionais de saúde a pré-diagnósticar possíveis pacientes com esquizofrenia para em seguida serem encaminhados a tratamentos adequados. O sistema fuzzy foi baseado em 5 sintomas da doença referenciados no DSM-5 (Manual de Diagnóstico e Estatístico de Transtornos Mentais) tendo como efeito o critério A e seus sintomas como variáveis de entrada: 
delírios, alucinações, discurso desoganizado, comportamento grosseiramente desorganizado ou catatônico e sintomas negativos.

[de Azevedo et al. 2019] desenvolveu um protótipo de um aplicativo de sistema especialista para auxiliar no diagnóstico da esquizofrenia com base nas diretrizes da CID10, capaz de auxiliar psiquiatras no diagnóstico esquizofrênico. Tal protótipo de aplicativo foi desenvolvido para a plataforma Android, disponível para uma variedade de dispositivos móveis, como smartphones e tablets.

[de Brito et al. 2020] desenvolveu um estudo através da implementação e análise das redes neurais Multilayer Perceptron (MLP) e Radial Basis Function Neural Network (RBF), objetivando comparar resultados baseados no treinamento, teste e classificação de crianças com/sem o transtorno do espectro autista (TEA), através da implementação de uma base de dados composta por 292 amostras de indivíduos de um banco de dados público.

Já [Fonseca 2019] propôs um modelo para auxiliar no diagnóstivo do transtorno bipolar, da depressão maior e da esquizofrenia, utilizando biomarcadores e características simples da população amostrada. Para o treinamento da rede neural artificial utilizou-se o OpenNN de distribuição livre. Obteve-se como resultado mais de $80 \%$ de acurácia nas classificações dos diagnósticos.

[Vergara 2018] propõe a aplicação de deep learning para classificação automática de imagens de ressonância magnética estrutural do cérebro e diagnóstico da esquizofrenia, produzindo métricas de desempenho como precisão, acurácia e sensibilidade relativos ao diagnóstico. O banco de dados utilizado contou com imagens de 87 indivíduos diagnosticados previamente com esquizofrenia e 85 indivíduos saudáveis de controle.

\section{Metodologia}

Para a criação do sistema fuzzy fez-se necessário tanto a utilização do Fuzzy Logic Toolbox do Matlab e o modelo de inferênca Takagi-Sugeno, quanto o I, que serviu para visualizar por linha de código o que estava sendo modelado. Foram geradas 729 regras de inferências, cada uma representando uma possível situação que um paciente possa enfrentar.

A Figura 1 demonstra a estrutura utilizada para a construção do sistema fuzzy, onde encontram-se 6 entradas, sendo que para cada entrada existe uma variável linguística e três valores que assume intervalos [0.0 e 0.39] leve, [0.40 e 0.69] moderado e [0.70 e 1] grave, ou seja, os níveis de gravidade para cada sintoma.

À Figura 2, o retângulo branco da esquerda apresenta as regras criadas e posteriormente o quadrado azul da direita representa as variáveis de saída, ou seja, as possíveis cinco saídas desejadas, que são: 0 Muito Baixo; 0.25 Baixo; 0.50 Médio; 0.75 Alto e 1 Muito Alto.

A Figura 3 demonstra a definição das regras, as mesmas selecionadas de azul foram escolhidas para a simulação com os pacientes fictícios. Pode-se observar que as regras selecionadas são 26, 145 e 571, as mesmas foram simuladas e apresentadas na Seção Resultados e Discurssões no capitulo 5 deste trabalho. As combinações das entradas e saídas desejadas formulam a possibilidade do risco do paciente ter esquizofrenia. 


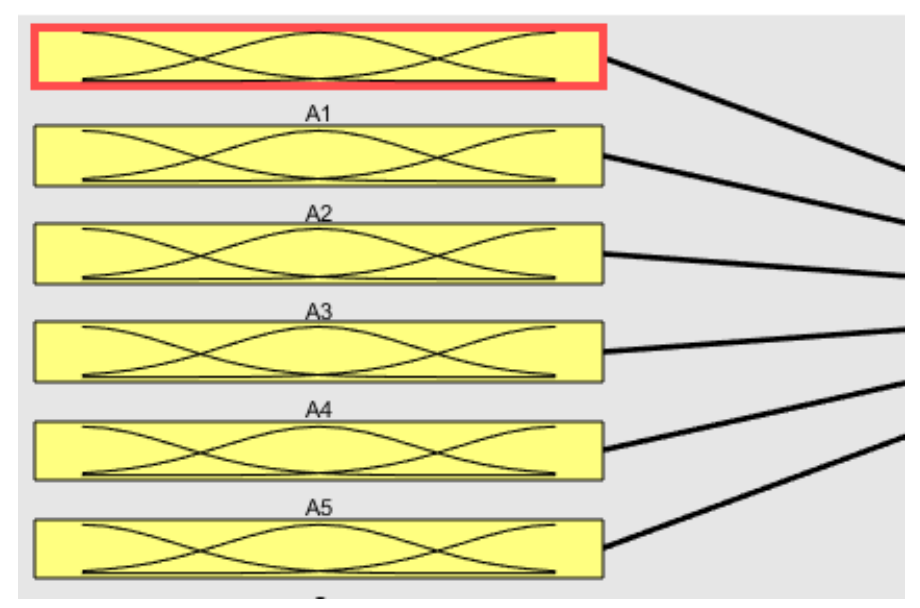

Figura 1. Estrutura do Sistema

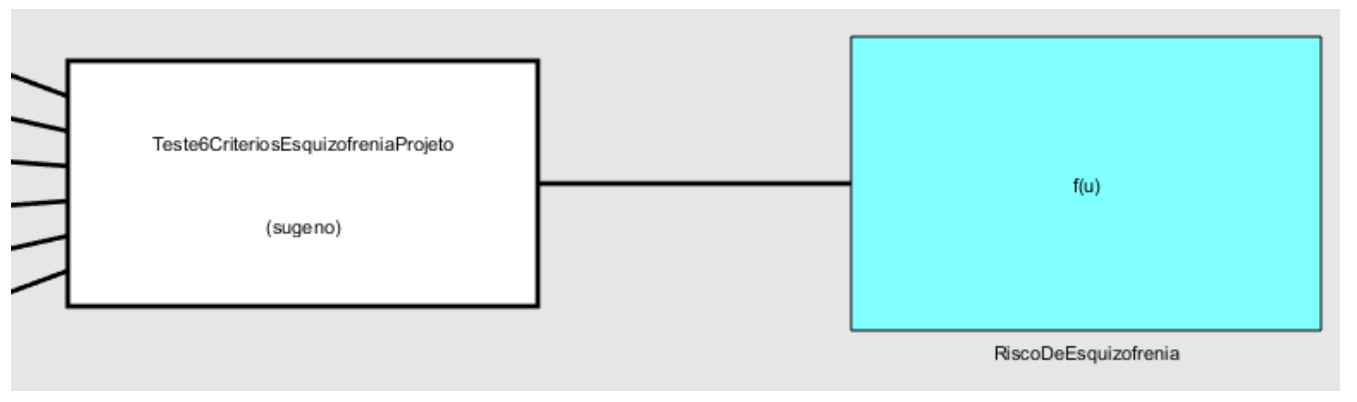

Figura 2. Regras e Saída

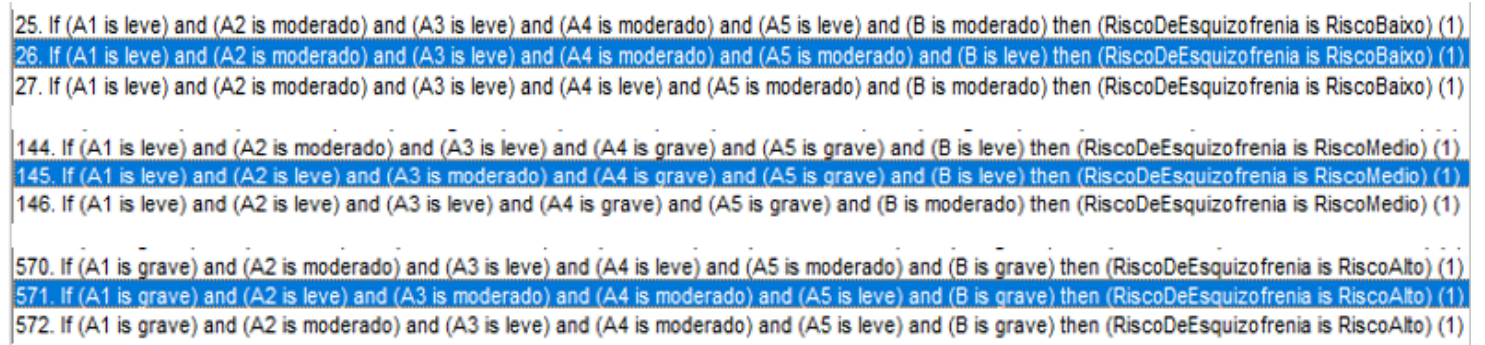

Figura 3. Regras de Inferência no Sistema Fuzzy

A Figura 4 demonstra uma parte do código fonte do sistema fuzzy, onde aparece sete linhas de códigos. O sistema, o nome do projeto, o tipo de inferência utilizada, a versão, o número de entradas, o número de saída e o número de regras geradas.

\section{Resultados e Discussões}

Foram realizados os testes com 10 pacientes fictícios e escolhidos três casos para demonstrar os resultados obtidos pelo sistema. Dentre os casos escolhidos contemplou-se três saídas possíveis: baixo, médio e alta.

No primeiro cenário o paciente fictício A possui 26 anos e apresenta os seguintes sintomas: delírios na faixa de 0.31 (leve); alucinações 0.54 (moderada); discurso desorganizado 0.28 (leve); comportamento grosseiramente desorganizado 0.48 (moderado); sintomas negativos 0.52 (moderado); relacionamento interpessoal/ autocuidado 0.12 (leve), 


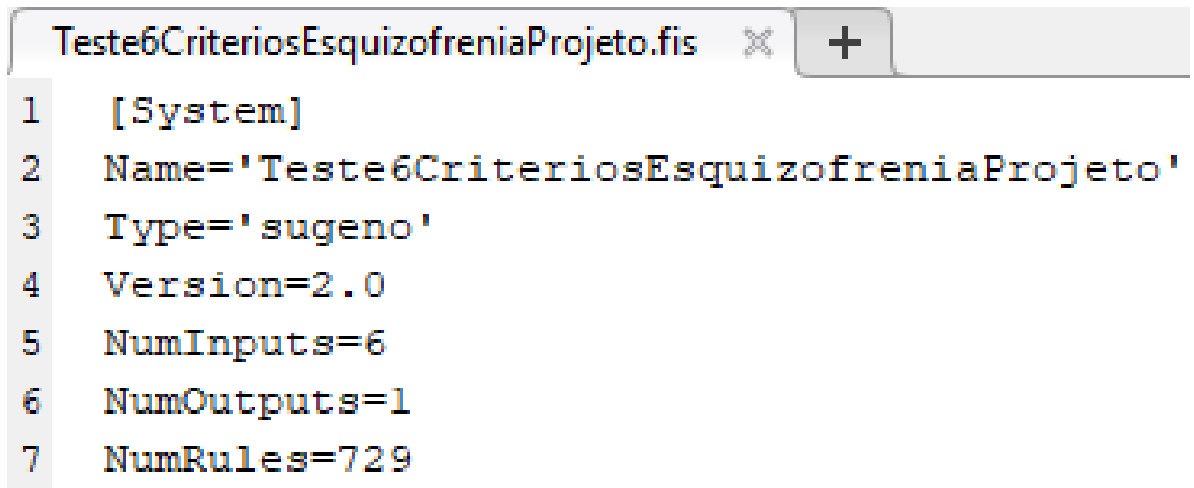

Figura 4. Script do Sistema Fuzzy

resultando em baixa possibilidade do paciente possuir a doença.

De acordo com a Figura 5, o resultado obtido foi 0.25 , que equivale ao baixo risco do paciente possuir a doença.
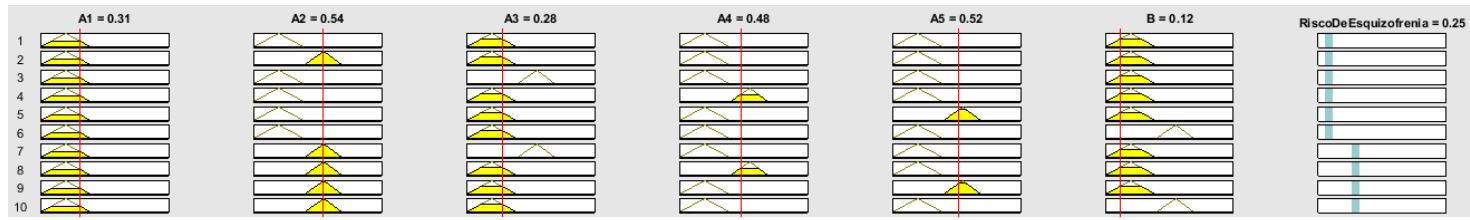

Figura 5. Resultados da Simulação com Paciente A

No segundo cenário o paciente fictício B possui 31 anos e apresenta: delírios na faixa de 0.21 (leve); alucinações 0.18 (leve); discurso desorganizado 0.61 (moderado); comportamento grosseiramente desorganizado 0.83 (grave); sintomas negativos 0.79 (grave); relacionamento interpessoal/ autocuidado 0.22 (leve), resultando em média possibilidade do paciente possuir a doença.

De acordo com a Figura 6, o resultado obtido foi 0.50, que equivale ao médio risco do paciente possuir a doença.
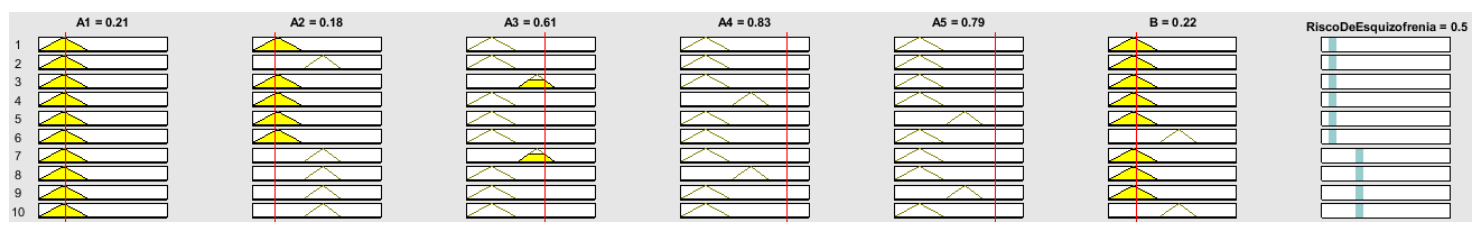

Figura 6. Resultados da Simulação com Paciente B

No terceiro cenário o paciente fictício C possui 37 anos e os sintomas estudados apresenta os seguintes resultados: delírios na faixa de 0.92 (grave); alucinações 0.12 (lve); discurso desorganizado 0.62 (moderado); comportamento grosseiramente desorganizado 0.57 (moderado); sintomas negativos 0.18 (leve); relacionamento interpessoal/ autocuidado 0.87 (grave), resultando em alta possibilidade do paciente possuir a doença.

De acordo com a Figura 7, o resultado obtido foi 0.75 , que equivale ao alto risco do paciente possuir a doença. 


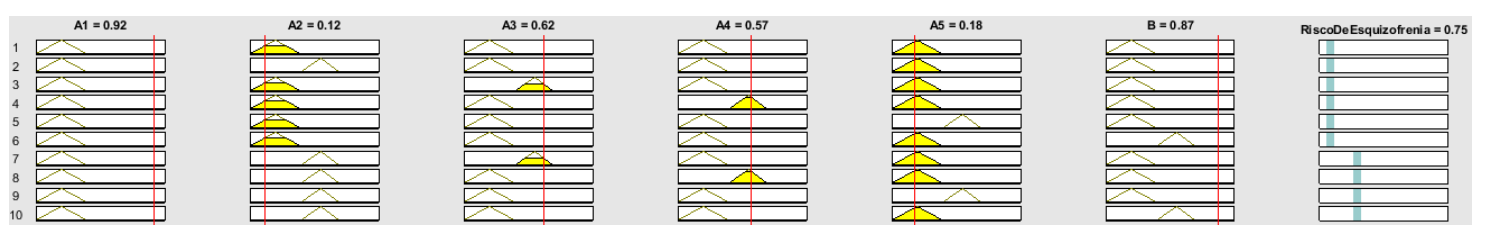

Figura 7. Resultados da Simulação com Paciente C

\section{Considerações Finais}

O presente estudo foi desenvolvido no intuito de corroborar com os profissionais da saúde, como dispositivo de pré-diagnóstico de pacientes possivelmente acometidos de esquizofrenia, de forma a diminuir o tempo com que os mesmos são encaminhados e a receberem o tratamento adequado, diminuindo, assim, a chance de sequelas.

Como trabalho futuro, sugere-se o incremento de mais variáveis de entrada de acordo com o DSM-5 e de regras de inferências para que o sistema torne-se mais robusto e com mais precisão. Sugere-se também que o sistema fuzzy passe pelo Comitê de Ética e Pesquisa e seja aplicado com dados de pacientes reais com o acompanhamento de um especialista em psiquiatria.

Por fim, recomenda-se a materialização desta pesquisa em um app ou site, também sob um eixo educacional, para educar e facilitar o auto-reconhecimento das famílias e pessoas que sofrem com a esquizofrenia.

\section{Referências}

Amorim, L. et al. (2018). Avaliação de funcionalidade em pacientes com esquizofrenia. Dissertação de mestrado, Universidade Federal de Santa Maria. Centro de Filosofia e Ciências Humanas, Programa de Pós-graduação em Psicologia.

Araújo, Á. C. and Neto, F. L. (2014). A nova classificação americana para os transtornos mentais-o dsm-5. Revista brasileira de terapia comportamental e cognitiva, 16(1):6782 .

Araujo, M. D. A., Moreira, L. Y. M. R., and de Brito, R. X. (2019). Modelo computacional com fuzzy como recurso auxiliador na predição da esquizofrenia em adultos. In Anais da VII Escola Regional de Computação Aplicada à Saúde, pages 199-204. SBC.

Association, A. P. et al. (2014). DSM-5: Manual diagnóstico e estatístico de transtornos mentais. Artmed Editora.

Behenck, A., da Silva, A. D., de Humerez, D. C., Mancia, J. R., and de Souza Padilha, M. I. C. (2011). A família frente ao processo de tratamento e reinternação do portador de esquizofrenia. Enfermagem em Foco, 2(4):210-214.

de Azevedo, M. S., Suárez, L. d. A. B., Caldas, M. T., de Sousa, M. N. A., de Sousa, T. B. d. S., Suárez, P. R., et al. (2019). Desenvolvimento de protótipo de sistema especialista para o auxílio no diagnóstico de esquizofrenia. Revista Brasileira de Educação e Saúde, 9(4).

de Brito, R. X., Fernandes, C. A. R., and Amora, M. A. B. (2020). Análise de desempenho com redes neurais artificiais, arquiteturas mlp e rbf para um problema 
de classificação de crianças com autismo. iSys-Revista Brasileira de Sistemas de Informação, 13(1):60-76.

Fonseca, M. B. (2019). Classificação do Transtorno Bipolar, Esquizofrenia e Depressão Utilizando Redes Neurais Artificiais. Tese de doutorado, Universidade Católica de Pelotas.

Lobo, L. C. (2018). Inteligência artificial, o futuro da medicina e a educação médica. Revista Brasileira de Educação Médica, 42(3):3-8.

Marro, A. A., Souza, A. d. C., Cavalcante, E. d. S., Bezerra, G. S., and NUNES, R. O. (2010). Lógica fuzzy: conceitos e aplicações. Natal: Universidade Federal do Rio Grande do Norte (UFRN).

Razzouk, D., Shirakawa, I., and Mari, J. d. J. (2000). Sistemas inteligentes no diagnóstico da esquizofrenia. Brazilian Journal of Psychiatry, 22:35-37.

Rosa, M. A. and Elkis, H. Adesão em esquizofrenia. Arquivos de Psiquiatria Clínica (S a o Paulo), 34:189-192.

Shirakawa, I. (2000). Aspectos gerais do manejo do tratamento de pacientes com esquizofrenia. Brazilian Journal of Psychiatry, 22:56-58.

Vergara, R. F. (2018). Detecção de alterações cerebrais anatômicas associadas à esquizofrenia com base em redes convolucionais aplicadas a imagens de ressonância magnética. Dissertação de mestrado, Universidade de Brasília. 